\title{
Nonconscious temporal cognition: Learning rhythms implicitly
}

\author{
JOANNA SALIDIS \\ Stanford University, Stanford, California
}

\begin{abstract}
Two experiments demonstrate that people can implicitly learn rhythms. Participants responded to a series of fast-paced beeps by pressing a key as soon as possible after each beep. They were not told that the duration $(180,450$, or $1,125 \mathrm{msec})$ between each keypress and the next beep was specified by a repeating sequence. In both experiments, participants responded significantly faster to predictable, sequenced timing than to random timing but did not show more knowledge of the sequence than did control participants on explicit memory measures. This dissociation was obtained even with an explicit memory test in Experiment 2 that maintained the same context and response metric as the implicit task to maximize the transfer of relevant knowledge. Implications for temporal cognition are discussed.
\end{abstract}

Music perception and performance are prototypical examples of human activities that rely on temporal cognition. Time has a clear and important role in music: Rhythm, the order and duration of notes and rests, is one of its two primary dimensions (Krumhansl, 2000). The demands that music perception and production make on temporal processing can be illustrated with a basic computational analysis. Perceiving and remembering rhythms requires segmenting rapid continuous auditory signals into meaningful units. Producing them requires precise control of individual effectors to maintain prescribed timing and sequential relationships. Although these temporal requirements are somewhat obvious in regard to playing an instrument or tapping a rhythm, their presence is less visible in other common human activities that utilize timing. For example, segmenting rapid signals into ordered constituent parts is omnipresent both in language perception and in receiving Morse code, a perceptual-motor skill. Similarly, speaking and tapping Morse are fundamentally exercises in rhythm production, since they require the maintenance of order and duration.

If there are common demands on temporal processing across skills, one might expect common solutions. Indeed, research suggests that many temporal processes may be shared across domains. Most fundamentally, numerous studies have demonstrated that interval estimation and production (manual tapping) share duration-dependent variability (Ivry \& Hazeltine, 1995; Ivry \& Keele, 1989; Keele, Pokorny, Corcos, \& Ivry, 1985; Treisman, Faulkner, \& Naisch, 1992). These results suggest that perceptual and

These experiments were part of a doctoral dissertation submitted by J.S. to the faculty of the University of Virginia. Portions of this research were presented at the 27th, 28th, and 29th annual meetings of the Society for Neuroscience, November 1997, 1998, and 1999. Thanks to Dan Willingham for advice and ideas. Correspondence should be addressed to J. Salidis, Department of Psychology, Stanford University, Jordan Hall, Building 420, Stanford, CA 94305 (e-mail: joanna@ psych.stanford.edu). motor timing use the same internal timing mechanism, or clock (for reviews, see Ivry, 1993, 1996). Common temporal processes may also be operative in language and other perceptual and motor skills. To be uniquely identified, some phonemes require the perception of frequency changes that occur over as little as $40 \mathrm{msec}$ (Tallal, 1980; Tallal, Miller, $\&$ Holly-Fitch, 1993). Impaired perception of such rapid changes may underlie the poor phonological skills seen in dyslexic and language-impaired children (Tallal et al., 1996; for a review, see Farmer \& Klein, 1995). These children are also impaired on other tasks requiring rapid temporal processing, such as rhythmic bimanual tapping and learning Morse code, suggesting common processes between verbal and nonverbal skills (Rudel, Denkla, \& Spalten, 1976; Schwartz \& Regan, 1996; Wolff, Michel, Ovrut, \& Drake, 1990).

Although parallels in temporal cognition are clear across several domains, different skills also have unique temporal requirements. One dimension on which skills differ is the degree to which temporal information is explicitly represented. Timing is an integral part of a musical score, for example, and musicians strive to replicate it ( $\mathrm{Pal}-$ mer, 1989). The same performers, however, are probably not consciously controlling the mechanics responsible for the precise timing of their skilled performance (Palmer, 1989). To an even greater degree, timing in skills such as somersaulting and juggling is unlikely to be explicitly taught, intentionally learned, or explicitly represented in any way, even though timing is highly consistent within and across performers (Bardy \& Laurent, 1998; Beek \& Turvey, 1992; Lee, Young, \& Rewt, 1992). Similarly, people do not have insight into the sequential timing required of their speech effectors to produce fluent speech.

Differences in the degree to which timing is consciously mediated among skills could reflect important differences in the cognitive and neural processes underlying temporal cognition. Such differences, illustrated in the distinction between implicit and explicit memory, have proved fruit- 
ful for the study of memory. Implicit memory, such as priming and skill learning, is the unintentional, nonconscious use of previously acquired information, measured through performance rather than by direct query (Graf \& Schacter, 1985). Functional and neural dissociations verify the distinction between implicit and explicit memory (for reviews, see Gabrieli, 1998; Roediger \& McDermott, 1993). The study of temporal cognition might similarly benefit from broadening the range of inquiry to include nonconscious temporal learning and memory.

Studies of the role of awareness in eye blink classical conditioning (EBCC) provide the only experimental evidence for nonconscioustemporal learning. In delay EBCC, a tone is followed by an air puff in the eye; the two stimuli coterminate. Participants learn the association between the tone and the puff so that an originally reflexive blink is elicited from the tone. Participants must also learn the interval between the onset of the tone and the onset of the puff in order to blink before the puff. Successful timing of the conditioned response is considered a hallmark of learning (Ivry, 1993; Perret, Ruiz, \& Mauk, 1993). Clark and Squire $(1998,1999)$ demonstrated that EBCC, in amnesic and normal participants, was not dependent on awareness of the relationship between the tone and puff, which suggests participantshad formed an implicit memory for the duration of the interval. Interestingly, however, awareness is required in a variation, trace EBCC (Clark \& Squire, 1998). In trace conditioning, a brief delay is inserted between the end of the tone and the beginning of the puff.

The goal of the present research was to determine whether normal young adults could implicitly, or unconsciously, learn rhythms. For this purpose, we adapted a sequence learning paradigm used to study implicit spatial sequence learning, the serial response time task (Nissen \& Bullemer, 1987). Amnesic participants show normal changes on performance measures (response times [RTs]) in the spatial version of this task, while failing to show explicit memory of the sequence on recall or recognition tests (Reber \& Squire, 1994, 1998). Neurologically intact participants can also learn the sequence in this task, without becoming aware of it (Mayr, 1996; Reed \& Johnson, 1994; Willingham, 1999; but see Shanks \& St. John, 1994).

To the participant, our task simply consisted of a series of fast-paced beeps; participants were to hit a response key as quickly as possible after each beep. The participants were not told that, for most of the experiment, the time that each beep occurred was determined by a continuous, repeating six-interval sequence. The interval between each response and the subsequent beep was 180 (1), 450 (2), or 1,125 (3) msec, depending on the present point in the sequence. For example, if the sequence was 121323 , and the participant had just responded to the third beep, then the next beep would occur 1,125 msec after the response.

The most important learning measure on this task is the difference in RT when the timing is predictable, due to the sequence, relative to when it is pseudo-random. When the timing is pseudo-random, the beeps still occur after one of the three intervals; however, the interval occurring at any given point is not predictable. If participants learn the sequence, they should respond faster when the timing is predictable than when it is not. Such response facilitation would indicate that participants precisely or categorically learned the intervals and the order in which they occurred. This measure reveals both types of learning because the timing of each signal and response, as with the elements of any rhythm, is jointly determined by sequence position and interval. In contrast to the specific information gained by this learning measure, decreases in RT over the course of the experiment, or when the timing is pseudorandom, could indicate general practice effects due to familiarity with the task, as well as learning the intervals.

This paper presents two experiments. Experiment 1 used the task described above to demonstrate implicit learning. To ensure that learning depended on implicit, as opposed to explicit, learning, we also measured participants' awareness of the sequence. In this explicit test, participants entered numbers, each representing an interval, into the computer in the same order that they believed the intervals had occurred in the repeating sequence. Participants then responded to a sequence of beeps following the timing that they had just entered. They were encouraged to repeat this process until they were satisfied that the produced sequence was the best match possible to the sequence they had experienced earlier. Experiment 2 introduced a new learning measure for the implicit task described above. As in Experiment 1, we measured rhythm learning as the relative facilitation of responses following sequenced relative to randomly ordered intervals. The second measure was the relative inhibition of a response following a "wrong" interval in the midst of the sequence relative to the same interval in a random context. Experiment 2 used a cued recall test that closely resembled the implicit task to measure awareness. Chance performance on the explicit tests in both experiments was determined from the scores of a second group of participants who were exposed only to randomly ordered intervals.

\section{EXPERIMENT 1}

\section{Method}

\section{Participants}

Participants were 48 undergraduate students from the University of Virginia who participated in return for course credit. Participants were randomly assigned to either the random or sequence group, with an equal number of participants in each group.

\section{Stimuli and Apparatus}

Data were collected on Apple IIci computers. Timing was accurate within 2 msec. Beeps were $392 \mathrm{~Hz}$ (83-msec duration). Responses, hitting the " $m$ " key on a standard keyboard, were made with the index finger of the dominant hand.

Each trial consisted of an empty interval that was 180 (1), 450 (2), or 1,125 (3) msec long, a beep and the response to the beep, in that order. There was no intertrial interval; the next trial's interval began immediately following the response to the prior trial. Trials were presented in blocks of 96 trials in a row. Rest breaks of $30 \mathrm{sec}$ followed each block. 
One of two target sequences, 121323 or 313212 , was randomly assigned to each participant in the sequence group. Blocks were either sequenced or random. In sequence blocks, the interval $(1,2$, or 3 ) for each trial was determined by the assigned sequence. In random blocks, the interval $(1,2$, or 3$)$ on each trial was randomly chosen, with the constraints that each interval would occur equally often and no interval would occur twice in a row.

\section{Procedure}

Implicit task. Participants were told that the purpose of the experiment was to study basic perceptual-motor processes. They were instructed to simply respond to the beep with a keypress as quickly as possible. They were also told to avoid pressing the key before they heard the beep. Feedback was limited to a message on the screen (interrupting the task for $2 \mathrm{sec}$ ) that informed participants that they had responded too soon, if they responded prior to the beep.

Participants in the sequence group responded to four sequence blocks, a random block, and a final sequence block. Participants in the random group responded to six random blocks.

Interview. Participants in both groups were interviewed immediately following the implicit task. They were informed that for most of the experiment, the timing of each beep was determined by a repeating sequence, like a rhythm. Participants were asked whether they had noticed this sequence. Regardless of the response, participants were also asked to indicate which of the last two blocks did not have the sequence in it. Finally, participants were told that despite what they were just told, they may or may not have been responding to a sequence. Participants were asked to rate their confidence that they had been responding to a sequence on a scale of 1 to 9 ( $1=d e f$ initely random, $5=$ no idea, and $9=$ definitely a sequence $)$.

Explicit test. Following the interview, participants were told that the sequence that they had responded to could have repeated after every 3,6 , or 12 trials. Participants were misled in this way to encourage them to include fragmentary knowledge, such as a sequence of only three intervals in a row. They were instructed to use the 1,2 , and 3 keys on the keyboard, which represented the short, middle, and long intervals, to enter 12 intervals in a row. Their goal was to replicate the interval order they had responded to in the implicit task. After entering 12 numbers, participants responded to 12 trials that used the interval order they just entered. Participants were encouraged to assess whether the sequence they had entered seemed right after responding to it. This procedure repeated until participants felt they had approximated the target sequence as well as they could. Participants also kept a record of their previous responses on a paper sheet. We encouraged participants to use this record to help them zero in on trials they wanted to change; this allowed them to avoid starting over for each new sequence they entered.

Participants were required to enter 12 numbers into the computer; if they only knew three or six intervals in a row, they were to repeat the same numbers or guess.

\section{Results and Discussion}

Data were analyzed separately for each interval because $\mathrm{RT}$ is a decreasing function of duration in simple response time tasks that include a range of intervals (for reviews, see Luce, 1986). This function reflects the fact that the probability that the signal will occur increases as the duration of the interval increases. Therefore, RTs to shorter intervals are longer than RTs to longer intervals.

Analyses were designed to measure sequence learning, including partial knowledge that could account for RT gains. Research on implicit sequence learning using spatial sequences indicates that triplets, or sequence knowledge of a position based on only the prior two positions, may be the primary unit learned (Howard \& Howard, 1997;
Jiminez, Mendez, \& Cleeremans, 1996). If participants primarily learned triplets rather than the entire sequence, then comparing performance between the random block and the sequence blocks would underestimate their learning. Participants might respond as fast to trials in the random block that could be predicted by sequence knowledge of the prior two trials as they would to trials in the sequence block. For example, if the sequence is 121323 , then a long interval followed by a medium interval specifies that the next interval will be long. Accordingly, if the triplet 323 occurs in the random block, then the RT to the final long interval would be dropped from the analysis because it is as predictable as a sequenced interval given knowledge of the triplet. Approximately 32 trials (33\%) were dropped from the random block for participants in the sequence group for this reason.

The Huyns-Feldt correction for repeated measures analysis of variance (ANOVA) was used to correct for violations of sphericity. Responses that occurred prior to the signal (anticipations) or more than $800 \mathrm{msec}$ following it were considered errors.

\section{Implicit task}

Errors. Fewer than $2 \%$ of the RTs were greater than $800 \mathrm{msec}$, and the number of these errors did not differ between the sequence $(M=0.72 \%, S D=0.69)$ and random $(M=1.5 \%, S D=3.2)$ groups $[t(46)=1.19, p>.2]$. The random group made more anticipative errors $(M=2.9 \%$, $S D=1.6)$ than the sequence group $(M=2.0 \%, S D=1.3)$ $[t(46)=2.32, p<.05]$.

RTs. Median RTs were calculated for each block and participant to use as summary scores for all analyses.

Figure 1 shows the mean RTs of the sequence and random groups for each block and interval. Two effects of sequence learning can be seen from this figure. First, the sequence group responded slower in the random block than in the sequence blocks. Second, the random group's RTs were slower than the sequence group's for each interval.

The first effect was tested with a repeated measures ANOVA with interval as a within-subjects factor and group as a between-subjects factor. The dependent variable was the difference between the median RT in the random block (Block 5) and the mean of the median RTs in Blocks 4 and 6 (called a learning score). For the sequence group, this measure reflects the cost of losing the sequence. For the random group, it reflects block to block variability. The main effect of group was significant $[F(1,46)=12.51$, $\left.M S_{\mathrm{e}}=2,630.38, p<.001\right]$; the sequence group's larger scores indicate a greater change in RT than would be expected from block to block variability. The interaction between group and interval was also significant $[F(2,92)=$ $\left.11.75, M S_{\mathrm{e}}=911.89, p<.001\right]$, but planned contrasts showed that sequence participants' scores were greater than random participants' for the short $[F(1,92)=110.17$, $\left.M S_{\mathrm{e}}=911.89, p<.01\right]$, medium $\left[F(1,92)=4.94, M S_{\mathrm{e}}=\right.$ $911.89, p<.05]$, and long $\left[F(1,92)=4.05, M S_{\mathrm{e}}=911.89\right.$, $p<.05]$ intervals. The main effect of interval was also reliable $[F(2,92)=14.42, p<.001]$. 


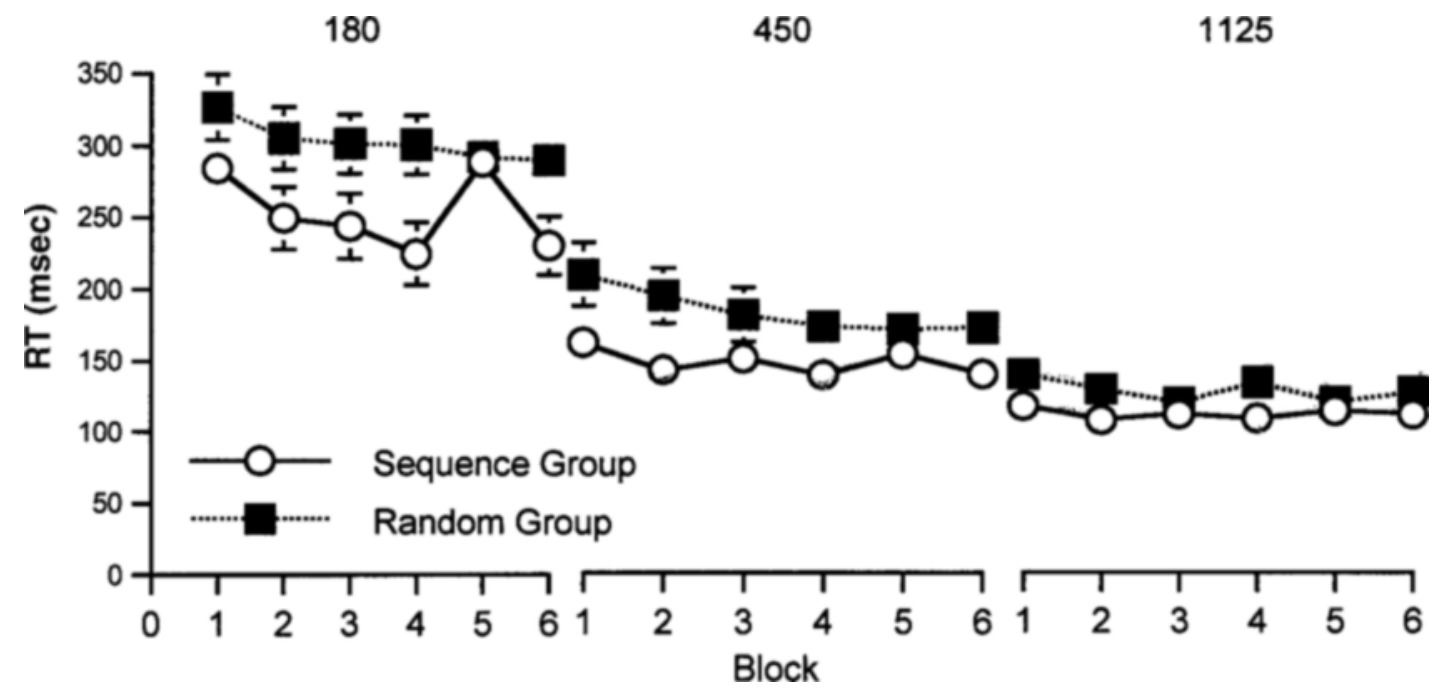

Figure 1. Mean response time (RT) by interval and group. The fifth block presented randomly ordered intervals to both groups. Error bars represent standard errors.

It is not clear from these results whether participants primarily learned triplets or a larger portion of the sequence. If the former, then including the trials that were dropped from the random block should decrease the learning scores. Including these trials decreases the short learning score by $2.54 \mathrm{msec}(4 \%)$, the medium by $2.67 \mathrm{msec}(22 \%)$, and the long by $1.42 \mathrm{msec}(68 \%)$. This finding suggests that learning the duration of only three intervals in a row can account for some of the demonstrated learning.

The second effect shown in Figure 1 is that the sequence group responded faster than the random group. A repeated measures ANOVA on the median RTs with block (1-6, excluding Block 5, which should not differ between the groups) and interval as within-subjects factors and group as a between-subjects factor confirmed this difference. The main effect of group was reliable $[F(1,46)=$ $\left.4.90, M S_{\mathrm{e}}=46,504.5, p<.05\right]$. The interaction between group and interval was also significant $[F(2,92)=4.28$, $\left.M S_{\mathrm{e}}=6,456.68, p<.05\right]$, but planned contrasts showed that the sequence group was significantly faster than the random group following the short $[F(1,92)=63.01$, $\left.M S_{\mathrm{e}}=6,456.68, p<.01\right]$, medium $[F(1,92)=20.12$, $\left.M S_{\mathrm{e}}=6,456.68, p<.01\right]$, and long $\left[F(1,92)=4.51, M S_{\mathrm{e}}=\right.$ $6,456.68, p<.05]$ intervals.

The main effect of interval was significant $[F(2,92)=$ $\left.52.41, M S_{\mathrm{e}}=6,456.68, p<.001\right]$, showing that RTs decreased as the intervals increased. The block $\times$ interval interaction was also significant $\left[F(8,368)=4.36, M S_{\mathrm{e}}=\right.$ $677.53, p<.001]$, as was the main effect of block $[F(4,184)$ $\left.=4.50, M S_{\mathrm{e}}=3,494.76, p<.01\right]$. The interaction between block and group was not significant $[F(4,184)<1]$, nor was the interaction between block, group, and interval $\left[F(8,368)=1.58, M S_{\mathrm{e}}=677.53\right]$.

One interesting feature of these results is that sequence learning, as measured by the learning scores and the overall difference in RT between the groups, decreased as the intervals increased. In fact, although significant, the difference between the groups' learning scores for the longest interval is quite small; as inspection of Figure 1 reveals, the effect partially relies on an inexplicable dip in the RTs of the random group. RT following both the sequenced and random intervals also decreased as the intervals increased. RTs are shortest following the long interval because the likelihood of stimulus occurrence increases with time (Kornblum, 1973; Näätänen, 1970). Similarly, the passage of time beyond $450 \mathrm{msec}$ categorically specifies the duration of the current interval as long. Knowledge derived from sequence learning may not be able to facilitate RTs further, resulting in a floor effect.

\section{Explicit knowledge}

Interview. More participants in the sequence group (19) said they had noticed the sequence after being told that there was one than did those in the random group (12) $\left[\chi^{2}(1)=4.46, p<.05\right]$. Those in the sequence group also gave higher ratings of confidence $(M=7.2, S E=0.26)$ that they had been responding to a sequence than did those in the random group $(M=5.9, S E=0.27)[t(46)=3.49$, $p<.01](1=$ definitely random, $5=$ no idea, and $9=$ def initely a sequence).

Although many participants noticed the sequence, most of them could not identify which of the last two blocks was random. Participants in the sequence group (17) were not more likely than those in the random group (16) to answer this question correctly $\left[\chi^{2}(1)<1\right]$.

Explicit test. Only the final production of the sequence on the explicit test was scored. The score was the number of triplets in the production that matched the target triplets. Target triplets were defined from the target sequence for 
each participant. For example, for the target sequence, 121323 , triplets were $121,213,132,323,231$, and 312 . Identical triplets in the production were counted only once. One of the two target sequences learned by the sequence participants was randomly assigned to each participant in the random condition. Each random participant's production was scored using this assigned sequence as the correct standard. Results showed that the sequence group did not recall more triplets $(M=1.92, S E=0.28)$ than the random group $(M=2.08, S E=0.28)[t(46)=-0.42, n . s$.$] .$

The results from this experiment demonstrate implicit temporal learning. Responses were facilitated following each of the three intervals when they occurred in a predictable order, indicating that participants learned to time their responses on the basis of the duration of at least two preceding intervals. Importantly, the results from the interview and explicit test show that these performance gains should not be attributed to explicit memory. Participants did not know which of the last two blocks was sequenced; nor could they produce more correct triplets than did the random group.

One caveat to these conclusions is that there was very little facilitation following the longest interval. This suggests that though participants learned the rhythm, they may not have learned the whole thing. They may have learned only the order of the shorter two intervals in the sequence. The alternative, investigated in Experiment 2, is that participants did learn the entire sequence, but learning was underestimated due to a floor effect.

\section{EXPERIMENT 2}

To determine whether learning in Experiment 1 was underestimated due to a floor effect, Experiment 2 included a second learning measure. Although sequence learning studies typically measure response facilitation, inhibition of incorrect responses is also a possible learning mechanism (Rauch et al., 1998). Accordingly, Experiment 2 measured response inhibition to out-of-order intervals within the context of the sequence. Out-of-order intervals were short or medium intervals that occurred in place of one third of the long intervals in a block of sequence trials. If participants expect to respond after a long interval, as predicted by the sequence, then responses to shorter intervals may be inhibited. Therefore, RTs following out-of-order intervals may be depressed relative to RTs following these same intervals within a neutral (random) context. Participants' RTs to randomly ordered intervals were measured in the neighboring blocks, which were half sequence trials and half random trials.

A second purpose of Experiment 2 was to replicate the result of response facilitation following sequenced short and medium intervals. Blocks that were half sequence and half random provide a measure of such learning, since responses should be faster during the sequenced portions of the block. A second measure may also be available from the block with the out-of-order intervals. Responses should be faster to in-order short and medium intervals than to out-of-order intervals, just as responses to sequence trials should be faster than those to random trials. However, if responses to out-of-order intervals are slower than those to the random intervals in the neighboring blocks, as predicted, then they may also be slower than responses to in-order intervals only because of inhibition due to learning the positions of the long intervals. To unambiguously attribute slower out-of-order responses to learning the positions of the shorter intervals, the out-oforder responses cannot differ from random responses.

The third purpose of Experiment 2 was to replicate the finding that learning occurred without awareness. Measuring awareness is difficult because it is necessary to ensure that the explicit test is sensitive to any knowledge, such as only parts of the sequence, that could result in performance gains (Shanks \& St. John, 1994). To increase sensitivity, the explicit test in this experiment maintained the same cues, context, and response metric as the implicit task. By changing only the instructions, to emphasize relying on memory rather than perception of the beeps to respond, we can assume that participants will use more explicit memory in the explicit test, if they have it, than they did in the implicit task (Merikle \& Reingold, 1991). In the explicit test, participants responded to blocks in which the order of each interval was determined by the sequence learned in the implicit task. On certain trials, however, rather than responding to the beep, participants were signaled to respond when they thought the beep should occur, on the basis of "what they had learned in the first part." RTs, on these trials, correspond to predictions about the duration of the current interval, since the responses are analogous to the stimuli on other trials. To demonstrate explicit knowledge, the RT on a prediction trial need not be veridical to the actual duration of the interval specified by the sequence for that trial. Instead, the RTs on prediction trials that should be short, for example, should categorically differ from the RTs on trials that should be medium or long.

\section{Method}

\section{Participants}

Sixty undergraduate students ( 16 men, 44 women) from the University of Virginia participated to partially fulfill a course requirement or for a payment of $\$ 6.00$. Participants were randomly assigned to either the random or the sequence group, with an equal number of participants in each group.

\section{Stimuli and Apparatus}

The stimuli and apparatus were identical to those of Experiment 1 except for the following changes. There were two types of blocks in the implicit task: learning and test. Their structure differed for the sequence and random groups. For the sequence group, learning blocks contained the following order of random and sequence trials: RRSS RRSS RRSS RRSS, where each R denotes six random trials and each $S$ denotes six sequence trials (one repetition of the sequence). The test block contained all sequence trials; however, three long intervals were replaced with the next-in-order intervals (short or medium) in every four repetitions of the sequence. The trials that occurred in place of the long intervals are called out-of-order trials because their duration is not predictable from the sequence. All other 
trials in the block are called in-order trials. The to-be-replaced long trials were randomly chosen with the constraint that there be three within every four sequence repetitions. For the random group, learning blocks contained all randomly ordered intervals. The test block also contained all randomly ordered intervals; however, long intervals were removed from the block using the same criteria as those used for the sequence group's test block.

The explicit test block was longer than the blocks in the implicit task; it had 192 trials. For the sequence group, the order of intervals was determined by the sequence learned in the implicit task. For the random group, the order of intervals was determined by a randomly assigned sequence. Most of the trials, called sequence trials, were exactly the same (interval, beep, response) as in the implicit task. However, there was a total of 12 "prediction" trials (four for each of the three intervals), which occurred in place of the sequence trials and had a different structure. Prediction trials consisted entirely of the word $G O$ ! in the center of the screen and the participant's response. Thus, the duration of the interval in a prediction trial, starting with the signal GO! and ending with the response, was determined by the participant, instead of being determined by the sequence. Each prediction trial was separated by a minimum of 12 trials to ensure that every possible "anchor" point (idiosyncratically designated start of the sequence) was available to the participants to maximize their chances for explicit recognition.

\section{Procedure}

Implicit task. As in Experiment 1, participants were told that the purpose of the experiment was to study basic perceptual and motor processes and that they should simply respond to every beep as quickly as possible. All participants responded to five learning blocks, the test block, and a final learning block. Rest breaks of $30 \mathrm{sec}$ separated each block. Feedback for anticipative errors was the only feedback provided-participants saw an unhappy face (1-in. diameter) in the center of the screen and heard an $u g h$ sound. The face was presented for $200 \mathrm{msec}$ before the experiment resumed.

Explicit test. Participants were not told anything about the temporal sequence. They were instructed to respond to each beep just as they had in the first part. In addition, they were told to expect the prediction trials and how they should respond to them. Participants were told that when they saw the word go, they should respond when they thought the next beep should occur, on the basis of what they had learned in the first part of the study, rather than waiting for it. They were told that their response replaced the beep for that trial. The task was interrupted if participants responded too slowly (more than 2,000 msec) on these trials, although participants were not told this in advance. The on-screen message said that they had responded too slowly and reminded them to respond when they thought the beep should occur, rather than waiting for it. The task resumed with a keypress.

\section{Results and Discussion}

Data were summarized and analyzed as in Experiment 1, except as noted. The random group's responses were divided into trial types analogous to the sequence groups' for analy sis purposes, even though they responded to all random trials. Therefore, for the random group, "random" and "sequence" trials were designated by their position in each block. This means, for example, that since the first 12 trials in each learning block were random and the 12th through 24th trials were sequenced for the sequence group, the first 12 trials were called random and the next 12 trials were called sequenced for the random group. Similarly, to make comparisons with the sequence group, the random group's trials were divided into in-order and out-oforder trials in the test block.
RTs greater than 2,000 msec were considered errors on the prediction trials in the explicit test.

\section{Implicit Task}

Errors. The percentage of RTs greater than $800 \mathrm{msec}$ was low and did not differ between the sequence $(M=$ $1.2 \%, S D=1.7)$ and random $(M=1.4 \%, S D=1.8)$ groups $[t(58)=0.26, p>.20]$. Nor were there any differences in the percentages of anticipative errors between trial types [sequence group: $\mathrm{S}(M=2.3 \%, S D=1.3), \mathrm{R}(M=2.3 \%, S D=$ $1.3)$ ] or groups [random group: $\mathrm{S}(M=2.4 \%, S D=1.4)$, $\mathrm{R}(M=2.6 \%, S D=1.7)$, all $t \mathrm{~s}<1]$.

Learning blocks. Learning scores (random minus sequence trials) were calculated from the means of the median RTs in the learning blocks. Figure 2 shows the learning scores for each group and interval. The figure shows that the sequence group responded faster to sequence than to random trials, but the random group responded faster to random than to sequence trials. The random group's results indicate that RTs to random trials capitalized on advantageous positions in the block because they were designated entirely by their position in the block. A repeated measures ANOVA on these data with interval as a withinsubjects factor and group as a between-subjects factor confirmed that the main effect of group was significant $\left[F(1,58)=11.53, M S_{\mathrm{e}}=381.92, p<.01\right]$. The interaction between group and interval was also significant $[F(2,116)=$ $\left.3.43, M S_{\mathrm{e}}=178.60, p<.05\right]$, but planned contrasts showed that the learning scores differed between the groups for the short $\left[F(1,116)=49.98, M S_{\mathrm{e}}=178.60, p<.01\right]$, medium $\left[F(1,116)=5.75, M S_{\mathrm{e}}=178.60, p<.05\right]$, and long $\left[F(1,116)=7.25, M S_{\mathrm{e}}=178.60, p<.01\right]$ intervals. The main effect of duration was not significant $[F(2,116)<1]$. This result replicates the finding from Experiment 1 that responses were facilitated for each of the three intervals when their order was predictable.

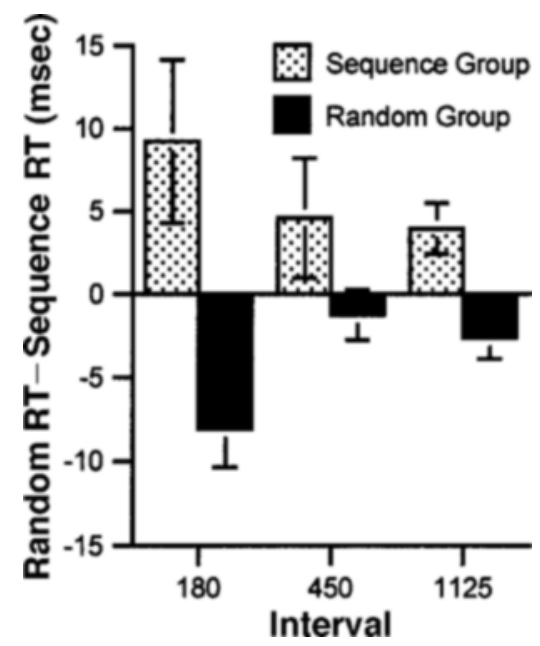

Figure 2. Difference in mean response time (RT) (random minus sequence) in the learning blocks for each group and interval. Error bars represent standard errors. 
Test block. The results for participants with less than five usable out-of-order trials per interval (due to anticipative errors) were not included in the analyses. After eliminating participants on this basis, there were 27 participants in the sequence group ( 3 dropped) and 26 in the random group (4 dropped).

Two learning scores were calculated for the test block analyses. The first was the median RT to the out-of-order trials minus the mean of the median RTs to random trials in Learning Blocks 5 and 7. The second learning score was the median RT to the out-of-order trials minus the median RT to the in-order trials.

The first question is whether responses to out-of-order trials were slower than those to random trials, a measure of learning-related response inhibition. A repeated measures ANOVA on the out-of-order minus random learning scores, with interval as a within-subjects factor and group as a between-subjects factor, showed that they were not greater for the sequence group $(M=19.15, S D=41.88)$ than for the random group $(M=23.76, S D=48.29)$ $[F(1,51)<1$ (main effect of group)]. The interaction between interval and group $\left[F(1,51)=2.44, M S_{\mathrm{e}}=1,441.61\right.$, $p>.10]$ was not significant, and the main effect of interval was marginal $\left[F(1,51)=3.20, M S_{\mathrm{e}}=1,441.61, p<\right.$ .10]. Because the scores did not differ between the groups, this analysis does not support the hypothesis that responses would be inhibited to beeps occurring sooner than predicted by the sequence.

The second question is whether responses to in-order trials were faster than responses to out-of-order trials. Since there was no learning-related inhibition for the outof-order trials, such a result can be unambiguously attributed to response facilitation of the in-order trials. Figure 3 shows the difference in RT between the out-of-order and the in-order trials for each interval and group. The difference between the trial types was larger for the sequence group, as confirmed by a repeated measures ANOVA on the learning scores $\left[F(1,51)=5.29, M S_{\mathrm{e}}=1,735.72, p<\right.$ .05 (main effect of group)]. The main effect of interval was marginal $\left[F(1,51)=3.43, M S_{\mathrm{e}}=1,825.65, p<.10\right]$. The interaction between interval and group was not significant $[F(1,51)<1]$. These results replicate the results of Experiment 1 , suggesting that participants learned when to expect the short and medium intervals.

\section{Explicit Test}

Errors. Neither the percentage of RTs greater than $800 \mathrm{msec}$ (sequence group: $M=3.0 \%, S D=2.5$; random group: $M=3.2 \%, S D=2.4$ ) nor anticipations (sequence group: $M=4.7 \%, S D=4.0$; random group: $M=6.8 \%$, $S D=6.5)$ differed between the groups on the sequence trials $(p s>.10)$. Nor did the groups have different percentages of RTs greater than $2,000 \mathrm{msec}$ on the prediction trials (sequence group: $M=2.2 \%, S D=4.3$; random group: $M=2.8 \%, S D=6.3, p>.20)$.

RTs. RTs on prediction trials measure explicit knowledge because they represent the participant's attempt to re-

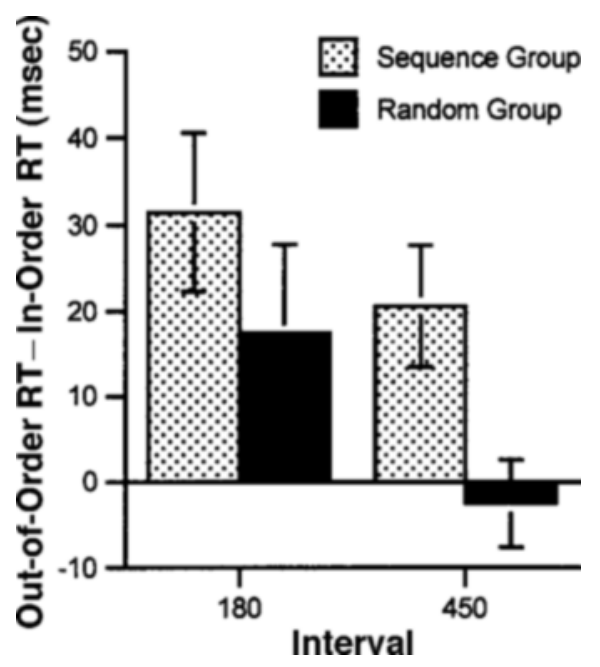

Figure 3. Difference in mean response time (RT) (out-of-order minus in-order) in the test block for each group and interval. Error bars represent standard errors.

produce the appropriate interval, as determined by the sequence, at that point. A repeated measures ANOVA on the median RTs with interval as a within-subjects factor and group as a between-subjectsfactor was conducted to determine whether participants in the sequence group adjusted their response timing to match the sequence-specified interval more than did participants in the random group. The interaction between interval and group was not significant $[F(2,116)<1]$, indicating that the sequence group did not differentiate between the intervals more than did the random group. Neither the main effect of interval $[F(2,116)=$ $1.30, p>.20]$ nor that of group $[F(1,58)<1]$ was significant. Means for the short, medium, and long intervals for the sequence group (with standard deviations in parentheses) were 697.03 (198.05), 734.53 (178.79), and 707.2 (199.49). Means for the random group were 723.67 (234.92), 763.27 (254.67), and 740.2 (219.34). These results suggest that the sequence group did not have conscious temporal knowledge because participants' predictions did not differ from those of the random group.

The explicit test also included a simultaneous measure, the sequence trial RTs, of implicit learning. An ANOVA on the median RTs with interval as a within-subjects factor and group as a between-subjects factor showed that the sequence group $(M=209.63, S D=30.03)$ was marginally faster than the random group $(M=232.16, S D=58.35)$ $\left[F(1,58)=3.53, M S_{\mathrm{e}}=7,940.6, p<.07\right.$ (main effect of group)]. Although this result misses the conventional cutoff, the difference between the groups occurs on top of a baseline of learning by the random group. Participants in that group were exposed to nearly 32 sequence repetitions in the explicit test. The interaction between interval and group was also marginal $\left[F(2,116)=2.93, M S_{\mathrm{e}}=544.17\right.$, $p<.08]$. The main effect of interval was significant $[F(2,116)=62.71, p<.01]$. 


\section{GENERAL DISCUSSION}

The results of the present research demonstrate for the first time that people can implicitly learn rhythms. In both experiments, participants were able to respond significantly faster to predictable, sequenced timing than to random timing. This response facilitation was demonstrated both within and between subjects, which controlled for factors such as trial type order. These experiments also show that participants learned without awareness. Experiment 2's explicit test, especially, was designed to be highly sensitive to any awareness of the rhythm. By maintaining the context and response metric of the implicit task, the explicit test maximized the potential for relevant knowledge, even if partial, to transfer. Furthermore, participants made the predictive judgments simultaneously with performing the sequences, showing a concurrent dissociation between their implicit (RTs faster than random) and explicit (predictions did not differ from random) knowledge.

These experiments also provide limited information about which aspects of the sequences were learned. Participants must have learned at least sequences of three elements because the sequences were structured so that the duration of each interval was predictable only from knowledge of the duration of the prior two. It is not clear, however, whether participants learned the order of the short intervals better than that of the long. Future research could investigate whether processes engaged by implicit temporal learning are tuned to intervals of less than $1 \mathrm{sec}$ or whether there is a bias toward shorter intervals in temporal learning paradigms not subject to floor effects. More broadly, research on whether implicit learning can be measured through response inhibition could be very fruitful for understanding its underlying mechanisms.

The present findings have implications for a broader understanding of temporal cognition. All previous research on temporal cognition has used as its standard conscious judgments and performance. It is not known how well, or whether, this research generalizes to nonconscious temporal phenomena. For example, it is well known that people learn and remember metrical rhythms better than nonmetrical ones (Povel, 1984; Povel \& Essens, 1985). Similarly, there is a strong bias in perception and production toward 1:2 interval ratios, such that people will distort intervals to fit this pattern (Collier \& Wright, 1995). These findings present a puzzle, since nonmetrical timing seems to exemplify timing in nonmusical activities such as speech and motor skills. The puzzle is that humans are believed to be especially suited to representing metrical rhythms, but common activities of daily life use nonmetrical timing while still conforming to exacting temporal standards.

A solution suggested by the present research is that timing in skills without explicit temporal goals may be implicitly learned. Explicit and implicit temporal learning processes may operate on different types of temporal phenomena, perhaps distinguished by their metric properties. Consistent with this proposal, distinct neural regions are active when people are recalling metrical ver- sus nonmetrical rhythms after brief delays (Sakai et al., 1999). Pilot experiments showed that nonmetrical timing, implemented by using response-to-stimulus rather than stimulus-to-stimulus intervals, was necessary to eliminate awareness of the rhythm. Rhythms of response-tostimulus intervals are inherently nonmetrical, even if the intervals are in a 1:2 ratio, because of the variable RT added to the duration of each produced interval. These observations suggest that nonmetrical timing may be the exclusive domain of implicit learning mechanisms. This solution offers an alternative to the view that timing in many skills without explicit temporal goals is not learned at all but is an emergent property of biomechanical, environmental, or task constraints (Beek, 1992; Beek \& Turvey, 1992; Kelso, 1997; Lee \& Young, 1986; Rumelhart \& Norman, 1982).

\section{REFERENCES}

BARDY, B., \& LAURENT, M. (1998). How is body orientation controlled during somersaulting? Journal of Experimental Psychology: Human Perception \& Performance, 24, 963-977.

BEEK, P. (1992). Inadequacies of the proportional duration model: Perspectives from a dynamical analysis of juggling. Human Movement Science, 11, 227-237.

BeEk,P., \& Turvey,M. (1992). Temporal patterning in cascade juggling. Journal of Experimental Psychology: Human Perception \& Performance, 18, 934-947.

Clark, R. E., \& Squire, L. R. (1998). Classical conditioning and brain systems: The role of awareness. Science, 280, 77-81.

Clark, R. E., \& Squire, L. R. (1999). Human eyeblink classical conditioning: Effects of manipulating awareness of the stimulus contingencies. Psychological Science, 10, 14-18.

Collier, G., \& Wright, C. (1995). Temporal rescaling of simple and complex ratios in rhy thmic tapping. Journal of Experimental Psychology: Human Perception \& Performance, 21, 602-627.

FARMER, M. E., \& KLEIN, R. M. (1995). The evidence for a temporal processing deficit linked to dyslexia: A review. Psychonomic Bulletin \& Review, 2, 460-493.

Gabrieli, J. D. E. (1998). Cognitive neuroscience of human memory. Annual Review of Psychology, 49, 87-115.

Graf, P., \& SCHACTER,D. L. (1985). Implicit and explicit memory for new associations in normal subjects and amnesic patients. Journal of Experimental Psychology: Learning, Memory, \& Cognition, 11, 501-511.

Howard, J. H., \& HowARD, D. V. (1997). Age differences in implicit learning of higher order dependencies in serial patterns. Psychology \& Aging, 12, 634-656.

IVRY, R. B. (1993). Cerebellar involvement in the explicit representation of temporal information. In P. Tallal, A. M. Galaburda, R. R. Llinas, \& C. von Euler (Eds.), Temporal information processing in the nervous system: Special reference to dyslexia and dysphasia (Annals of the New York Academy of Sciences, Vol. 682, pp. 214-230). New York: New York Academy of Sciences.

IVRY, R. B. (1996). The representation of temporal information in perception and motor control. Current Opinion in Neurobiology, 6, 851-857.

IV RY, R. B., \& Hazeltine, R. E. (1995). The perception and production of temporal intervals across a range of durations: Evidence for a common timing mechanism. Journal of Experimental Psychology: Human Perception \& Performance, 21, 1-12.

IVRY, R. B., \& KEELE, S. W. (1989). Timing functions of the cerebellum. Journal of Cognitive Neuroscience, 1, 134-150.

Jiminez, L., Mendez, C., \& Cleeremans, A. (1996). Comparing direct and indirect measures of sequence learning. Journal of Experimental Psychology: Learning, Memory, \& Cognition, 22, 948-969.

Keele, S. W., Pokorny, R. A., Corcos, D. M., \& Ivry, R. [B.] (1985) Do perception and motor production share common timing mechanisms: A correlational analysis. Acta Psychologica, 60, 173-191. 
Kelso, J. (1997). Relative timing in brain and behavior: Some observations about the generalized motor program and self-organized coordination dynamics. Human Movement Science, 16, 453-460.

Kornblum, S. (1973). Simple reaction time as a race between signal detection and time estimation: A paradigm and model. Perception \& Psychophysics, 13, 108-112.

Krumhansl, C. L. (2000). Rhythm and pitch in music cognition. Psychological Bulletin, 126, 159-179.

LeE, D., \& Young, D. (1986). Gearing action to the environment. In H. Heuer \& C. Fromm (Eds.), Generation and modulation of action patterns (pp. 217-230). Berlin: Springer-Verlag.

LeE, D., Young, D., \& Rewt, D. (1992). How do somersaulters land on their feet? Journal of Experimental Psychology: Human Perception \& Performance, 18, 1195-1202.

LUCE, R. D. (1986). Response times: Their role in inferring elementary mental organization. New York: Oxford University Press.

MAYr, U. (1996). Spatial attention and implicit sequence learning: Evidence for independent learning of spatial and nonspatial sequences Journal of Experimental Psychology: Learning, Memory, \& Cognition, 22, 350-364.

Merikle, P. M., \& Reingold, E. M. (1991). Comparing direct (explicit) and indirect (implicit) measures to study unconscious memory. Journal of Experimental Psychology: Learning, Memory, \& Cognition, 17, 224-233.

NÄÄTÄNEN, R. (1970). The diminishing time-uncertainty with the lapse of time after the warning signal in reaction-time experiments with varying fore-periods. Acta Psychologica, 34, 399-419.

NisSEN, M. J., \& BULlEMER,P. (1987). Attentional requirements of learning: Evidence from performance measures. Cognitive Psychology, 19, $1-32$.

Palmer, C. (1989). Mapping musical thought to musical performance. Journal of Experimental Psychology: Human Perception \& Performance, 15, 331-346.

Perret, S. P., Ruiz, B. P., \& Mauk, M. D. (1993). Cerebellar cortex lesions disrupt learning dependent timing of conditioned eyelid responses. Journal of Neuroscience, 13, 1708-1718.

Povel, D. J. (1984). A theoretical framework for rhythm perception. Psychological Research, 45, 315-337.

Povel, D. J., \& Essens, P. (1985). Perception of temporal patterns. Music Perception, 2, 411-440.

Rauch, S. L., Whalen, P. J., Curran, T., McInerney, S., Heckers, S., \& SAvage, C. R. (1998). Thalamic deactivation during early implicit learning: A functional MRI study. NeuroReport, 9, 865-870.

Reber, P. J., \& SQuire, L. R. (1994). Parallel brain systems for learning with and without awareness. Learning \& Memory, 1, 217-229.

REber, P. J., \& Squire, L. R. (1998). Encapsulation of implicit and explicit memory in sequence learning. Journal of Cognitive Neuroscience, 10, 248-263.
ReED, J., \& JoHnson, P. (1994). Assessing implicit learning with indirect tests: Determining what is learned about sequence structure. Journal of Experimental Psychology: Learning, Memory, \& Cognition, 20, 584-594.

Roediger, H. L., III, \& McDermott, K. B. (1993). Implicit memory in normal human subjects. In H. Spinnler \& F. Boller (Eds.), Handbook of neuropsychology (Vol. 8, pp. 63-131). Amsterdam: Elsevier.

Rudel, R. G., Denkla, M. B., \& Spalten, E. (1976). Paired associate learning of Morse code and Braille letter names by dyslexic and normal children. Cortex, 12, 61-70.

Rumelhart, D. E., \& Norman, D. A. (1982). Simulating a skilled typist: A study of skilled cognitive-motor performance. Cognitive Science, $\mathbf{6}$, 1-36.

Sakai, K., Hikosaka, O., Miy uchi, S., Takino, R., Tamada, T., Iwata, N., \& Nielsen, M. (1999). Neural representation of a rhythm depends on its interval ratio. Journal of Neuroscience, 19, 10074-10081.

Schwartz, M., \& Regan, V. (1996). Sequencing, timing, and rate relationships between language and motor skill in children with receptive language delay. Developmental Neuropsychology, 12, 255-270.

Shanks, D. R., \& ST. John, M. F. (1994). Characteristics of dissociable human learning systems. Behavioral \& Brain Sciences, 17, 367-447.

TALlal, P. (1980). Auditory temporal perception, phonics, and reading disabilities in children. Brain \& Language, 9, 182-198.

Tallal, P., Miller, S. L., Bedi, G., Byma, G., Wang, X., Nagarajan, S., Schreiner, C., Jenkins, W., \& Merzenich, M. (1996). Language comprehension in language-learning impaired children improved with acoustically modified speech. Science, 271, 81-84.

Tallal, P., Miller, S. L., \& Holly-Fitch, R. (1993). Neurobiological basis of speech: A case for the preeminence of temporal processing. In P. Tallal, A. M. Galaburda, R. R. Llinas, \& C. von Euler (Eds.), Temporal information processing in the nervous system: Special reference to dyslexia and dysphasia (Annals of the New York Academy of Sciences, Vol. 682, pp. 27-47). New York: New York Academy of Sciences.

Treisman, M., Faulkner, A., \& Naisch, P. L. N. (1992). On the relation between time perception and the timing of motor action: Evidence for a temporal oscillator controlling the timing of movement. Quarterly Journal of Experimental Psychology, 45A, 235-263.

Willingham, D. B. (1999). Implicit motor sequence learning is not purely perceptual. Memory \& Cognition, 27, 561-572.

Wolff, P. H., Michel, G. F., Ovrut, M., \& Drake, C. (1990). Rate and timing precision of motor coordination in developmental dyslexia. $\mathrm{De}$ velopmental Psychology, 26, 349-359.

(Manuscript received June 24, 1998; revision accepted for publication July 17, 2001.) 